$\xi=$

\title{
Optical properties and electronic polarizability of boron-antimonide semiconductor
}

\author{
Salah Daoud ${ }^{1}$, Abdelhakim latreche ${ }^{2} *$ \\ ${ }^{1}$ Laboratory of Materials and Electronic Systems (LMSE), Faculty of Sciences and Technology, \\ Mohamed Elbachir El Ibrahimi,Bordj Bou Arreridj University, Bordj Bou Arreridj (34000), Algeria \\ ${ }^{2}$ Département des Sciences de la matière, Université Mohamed Elbachir El Ibrahimi de Bordj Bou Arreridj, \\ Bordj Bou Arreridj (34000), Algérie \\ *Corresponding author E-mail: hlat26@yahoo.fr
}

\begin{abstract}
The high-frequency and static dielectric constants, the reflex index, the total optical electronegativity difference, the bulk modulus, the micro-hardness, the plasmon energy and the electronic polarizability of cubic zincblende boron-antimonide semiconductor have been estimated by using some empirical formulas. These parameters are analyzed by comparing them against the available experimental and theoretical data. In general, our obtained results agree well with other theoretical data from the literature.
\end{abstract}

Keywords: High-Frequency and Static Dielectric Constants; BSB Semiconductor Material; Cubic Zincblende Binary Compounds.

\section{Introduction}

Because of the technological importance of III-V semiconductors, their optical and electronic properties have been the subject with a great interest in both experiment and theoretical researches. Optical and electronic properties of semiconductor materials are important parameters for elements, compounds and alloys used in solar cell and optoelectronic devices manufacturing [1].

In order to study the optoelectronic properties such as highfrequency refractive index, optical susceptibility, electronic polarizability and crystal ionicity of some cubic zincblende and wurtzite type structured binary compounds, Yadav et al. [2] have used the plasma oscillation theory of solids formalism. They found that the high-frequency refractive index, the optical susceptibility, electronic polarizability and crystal ionicity of both cubic zinc blende, and wurtzite type structure compounds exhibit a linear relationship when they are plotted to a log-log scale as against the plasmon energy.

Using a new approach based on the concept of ionic charge theory, the lattice thermal conductivity and the bulk modulus of III-V and II-VI semiconductor materials were studied by Verma et al. [3] They found that the lattice thermal conductivity of these semiconductors exhibits also a linear relationship when it is plotted on a $\log$-log scale against the nearest-neighbor distance.

Bouhafs et al. [4] have used an ab initio calculation to study the electronic properties of BN, BAs and BSb compounds. They have found that the electronic properties of these semiconductor materials are different from those of other III-V compounds (the direct band gap pressure coefficient is nearly independent of the anion substitutions).

In our recent work [5-7], several physical quantities such as: cohesive energy, elastic constants, Kleinman parameter, Cauchy's discrepancy in elastic constant, Cauchy's coefficient, Born ratio, Knoop micro hardness, homopolar and heteropolar energies, the bonding-anti bonding energy gap, the covalency and the sound velocity of BSb semiconductor were studied.

In another recent work [8], we have established a quantitative form of the linear correlation between the high-frequency and static dielectric constants in $\mathrm{A}^{\mathrm{N}} \mathrm{B}^{8-\mathrm{N}}(\mathrm{N}=2,3)$ tetrahedral semiconductors, where we found a quasi-linear relationship between the high-frequency and static dielectric constants for II-VI and III$\mathrm{V}$ binary materials. Using $\mathrm{Ab}$ initio calculations, Yao and coworkers [9] have studied the electronic and phononic properties of bulk boron-antimonide semiconductor. They found that boronantimonide semiconductor can be a good material candidate with low-cost and easily processed, for hot carrier absorbers (HCA) in high-efficiency third generation solar cell. In this work, we would like to add some investigations, mainly on the optical and electronic properties of one III-V binary materials (BSb semiconductor). We calculate the high-frequency and static dielectric constants $\left(\varepsilon_{\infty}\right.$ and $\left.\varepsilon_{0}\right)$ of Zinc-Blende BSb Semiconductor by using the Verma's formula [1] and determine the corresponding reflex index, the total optical electronegativity difference, the bulk modulus, the micro-hardness, the plasmon energy, and the electronic polarizability. The new results are compared with other experiment and theoretical results.

\section{Theory, results and discussion}

\subsection{High-frequency and static dielectric constants}

For several III-V and II-VI types binary semiconductors with cubic zincblende structure; an efficient formula relates highfrequency $\left(\varepsilon_{\infty}\right)$ and static $\left(\varepsilon_{0}\right)$ dielectric constants with the ionic charges of the anion and the cation of compound were established by Verma et al. [1]. It is given as follows [1]:

$$
\varepsilon=1 /\left[A-S\left(Z_{a} Z_{c} Z_{a v} \times 0.25\right)\right]
$$


Where $\varepsilon$ indicates either $\varepsilon_{\infty}$ or $\varepsilon_{0}, Z_{\mathrm{a}}$ and $\mathrm{Z}_{\mathrm{c}}$ are the ionic charges on the anion and the cation, respectively, $Z_{\mathrm{av}}$ is the average atomic number of constituent atoms, and $\mathrm{A}$ and $\mathrm{S}$ are two constants. The values of the constants $A$ and $S$ are: $0.19308 \pm 0.01024$ and $0.0012626 \pm 1.53552 \times 10^{-4}$ for high-frequency dielectric constant $\left(\varepsilon_{\infty}\right)$, and $0.12954 \pm 0.00281$ and $6.79659 \times 10^{-4} \pm 4.21458 \times 10^{-5}$ for the static dielectric constant $\left(\varepsilon_{0}\right)$, respectively [1]. Thus, the new values of $\varepsilon_{\infty}$ and $\varepsilon_{0}$ of $B S b$ semiconductor can be calculated by replacing the corresponding values of $\left(\mathrm{Z}_{\mathrm{a}} \cdot \mathrm{Z}_{\mathrm{c}}\right)$ and $\mathrm{Z}_{\mathrm{av}}$ in Eq. (1), and they were found to be 8.8 and 11.53 respectively. Our calculated values of $\varepsilon_{\infty}$ and $\varepsilon_{0}$ are listed in Table 1 and compared with the available experimental [10] and theoretical [11-16] data in the literature. At given optical frequency, the dielectric constant $\varepsilon$ and the refractive index $\mathrm{n}$ are related with the following expression [12], [17]

$\mathrm{n}=\sqrt{\varepsilon}$

So with consequence the high-frequency and static (zerofrequency) reflex index $\left(\mathrm{n}_{\infty}, \mathrm{n}_{0}\right)$ are related to the high-frequency and static dielectric constants $\left(\varepsilon_{\infty}, \varepsilon_{0}\right)$ by the formula of Eq. (2). Our calculated values of $\mathrm{n}_{\infty}$ and $\mathrm{n}_{0}$ are also listed in Table 1 and compared with the available experimental [10] and theoretical [1116] data in the literature.

Table 1: High-Frequency ( $\left.\varepsilon_{\infty}\right)$, Static Dielectric Constants, $\left(\varepsilon_{0}\right)$, and Static Reflex Index $\left(\mathrm{n}_{\infty}, \mathrm{n}_{0}\right)$ of Zinc-Blende Bsb Semiconductor are in Comparison with Available Data of the Literature [10-16]

\begin{tabular}{lllll}
\hline Parameter & $\varepsilon_{\infty}$ & $\varepsilon_{0}$ & $\mathrm{n}_{\infty}$ & $\mathrm{n}_{0}$ \\
\hline This work & 8.8 & 11.53 & 2.97 & 3.40 \\
Ref. [10] & $16.77-19.28$ & - & $4.0-4.5$ & - \\
Ref. [11] & - & 6.37 & - & 2.52 \\
Ref. [12] & - & 11.17 & - & 3.30 \\
Ref. [13] GGA-PBE & - & 11.19 & - & 3.35 \\
Ref. [13] mBJ-GGA & - & 8.47 & - & 2.91 \\
Ref. [14] & 11.3 & - & - & - \\
Ref. [15] & 10.72 & - & - & - \\
Ref. [16] & - & - & - & 3.30 \\
\hline
\end{tabular}

As can be seen from Table 1 , our calculated values; $\varepsilon_{\infty}, \varepsilon_{0}, \mathrm{n}_{\infty}$, and $\mathrm{n}_{0}$ are generally in reasonable agreement with the previously theoretical data reported in Refs. [11-16], where for example, the deviation between our value (3.40) of $n_{0}$ and that (3.35) of the Ref [13] GGA-PBE is only about $1.5 \%$.

However, it is found that the experimental data [10] are very higher than our results, and all theoretical data reported in Refs. [1116]. The difference between our results and the theoretical values reported in Refs [11-16] and also with the experimental one reported in Ref. [10] is mainly due to the different expressions proposed to calculate these parameters.

The total optical electronegativity difference $\Delta X^{*}$ and the highfrequency dielectric constant $\varepsilon_{\infty}$ are related by the following empirical formula [17]

$$
\varepsilon=n^{2}=\left[-\ln \left(0.012 \Delta \mathrm{X}^{*}\right)\right]^{2}
$$

The total optical electronegativity difference $\Delta X *$ is related to the energy gap, $\mathrm{Eg}$ by the following expression: $7\left[1 \Delta \mathrm{X}^{*}=O .268 \mathrm{Eg}\right]$. Using the experimental energy gap $\mathrm{E}_{\mathrm{g}}=$ $0.55 \mathrm{eV}$ [10] of thin films cubic zincblende boron-antimonide semiconductor, the total optical electronegativity difference $\Delta X^{*}$ of this material is found to be 0.1474 . Replacing this value in the empirical formula of Eq. (3), a value of $\varepsilon_{\infty}=6.34$ was obtained, which consists well with our theoretical value (8.8) obtained in this work.

Anani et al. [18] reported also that, the refractive index of semiconductor is totally depending on its band gap. They have proposed a linear relationship between the refractive-index and the energy gap $E_{g}$, it is given by the following formula [18], [19]:
$\mathrm{n}=(17-\mathrm{Eg}) / 5$

By using the value of $E_{g}=0.55 \mathrm{eV}$ [10], the value of refractive index $\mathrm{n}$ of this compound obtained from the relation of Eq.(4) is found to be 3.29, which is relatively higher than our results (2.97), and lower than the value (4.0) measured by Das et al. [10].

\subsection{Bulk modulus, Micro-hardness and plasmon energy}

In this part of the present work, and in order to estimate the bulk modulus $\mathrm{B}$, the microhardness $\mathrm{H}$, and the plasmon energy $\hbar \omega_{\mathrm{p}}$ of $\mathrm{BSb}$ compound at room temperature, we use three empirical equations, which have been proposed by Reddy et al. [20]. The refractive index relates to the bulk modulus, microhardness, and plasmon energy for some groups II-VI and III-V semiconductors by the following relations [19], [20]:

$$
\begin{aligned}
\mathrm{B}(\mathrm{GPa}) & =\mathrm{K}_{1} \exp \left(\mathrm{K}_{2} \mathrm{n}\right)-\mathrm{K}_{3} \\
\mathrm{H}(\mathrm{GPa}) & =\mathrm{K}_{3} \exp \left(\mathrm{K}_{4} \mathrm{n}\right)-\mathrm{K}_{5} \\
\hbar \omega_{\mathrm{p}}(\mathrm{eV}) & =\mathrm{K}_{6} \exp \left(\mathrm{K}_{7 \mathrm{n}}\right)
\end{aligned}
$$

The relevant values of the constants $\mathrm{K}_{1}, \mathrm{~K}_{2}, \mathrm{~K}_{3}, \mathrm{~K}_{4}, \mathrm{~K}_{5}, \mathrm{~K}_{6}$, and $\mathrm{K}_{7}$ for III-V semiconductors are: $648.89,-0.3546,104.953,-0.3546$, $26.82,47.924$, and -0.3546 , respectively [20].

Replacing these values and our obtained value of the refractive index $\mathrm{n}=2.97$ in the empirical formulas of Eqs. (5), (6) and (7), the new values of the bulk modulus, the microhardness, and the plasmon energy of BSb compound are given in Table 2 and compared with the available theoretical data [5], [6], [21].

Table 2: Bulk Modulus, Microhardness, and Plasmon Energy of ZincBlende Bsb Semiconductor, in Comparison with Available Data Of The Literature [5], [6], [21].

\begin{tabular}{llll}
\hline Parameter & $\mathrm{B}(\mathrm{GPa})$ & $\mathrm{H}(\mathrm{GPa})$ & $\hbar \omega_{\mathrm{p}}(\mathrm{eV})$ \\
\hline This work & 121.4 & 9.79 & 16.72 \\
Ref. [5] & 110.1 & - & - \\
Ref. [6] & 106.8 and 108 & 9.73 and 11.54 & - \\
Ref. [21] & From 105.08 & From 11.3 & From 17.41 \\
& to 146.16 & to 16.51 & to 19.79 \\
\hline
\end{tabular}

It can be seen, that our calculated values of bulk modulus, microhardness, and plasmon energy of zinc-blende BSb semiconductor are in good agreement with the other theoretical calculations [5], [6], [21]; where for example the deviation of our value (9.79 GPa) of the microhardness from the calculated value $(9.73 \mathrm{GPa})$ reported in our previous work [6] is only about: $0.62 \%$.

\subsection{Electronic polarizability}

If we know the refractive index $\mathrm{n}$ and the crystal density $\mathrm{g}$ of any crystalline material, it's possible to predict the electronic polarizability $\alpha_{p}$ by using the following formula [22]:

$\alpha_{p}=\frac{\left(n^{2}-1\right) \cdot M \cdot 10^{-24}}{\left(n^{2}+2\right) \cdot g \cdot(2 \cdot 53)}$

Where, $\mathrm{n}$ is refractive index, $\mathrm{M}$ is the molecular weight of the material and $\mathrm{g}$ is the crystal density.

The crystal density $g$ is one of important quantity in solid state physic. In cubic zinc-blende structure, the X-ray crystal density g is given as follow [5], [23-25]: $\mathrm{g}=4 \mathrm{M} / \mathrm{N}_{\mathrm{AV}}$, where $\mathrm{M}$ is the molecular weight (for $\mathrm{BSb}$ compound, $\mathrm{M}=132.571 \mathrm{uma}$ ), $\mathrm{N}_{\mathrm{A}}$ is the Avogadro number $\left(\mathrm{N}_{\mathrm{A}}=6.022 \times 10^{23} \mathrm{~mol}^{-1}\right)$, and $\mathrm{V}$ is the volume.

Using the experimental lattice constant $\mathrm{a}=5.30 \AA$ 年easured by Das et al. [10] of thin films cubic zincblende BSb semiconductor, the crystal density of BSb compound is found to be $5.915 \mathrm{~g} / \mathrm{cm}^{3}$, which is relatively smaller than the value $6.37 \mathrm{~g} / \mathrm{cm}^{3}$ obtained from the local density approximation (LDA) [5]. 
Replacing our obtained value of the refractive index $n=2.97$, the molecular weight $\mathrm{M}=132.571$ uma for $\mathrm{BSb}$ compound and the $\mathrm{X}$ ray crystal density $\mathrm{g}=5.915 \mathrm{~g} / \mathrm{cm}^{3}$ in the empirical formula of Eq. (8), the value of the electronic polarizability $\alpha_{p}$ is found to be $6.40 \AA^{3}$. This value is relatively higher than the value $5.40 \AA^{3} \mathrm{ob}$ tained by Verma et al. [26]. Our value is also slightely higher than the values $5.07-5.63 \AA^{3}$ obtained by Shaileshkumar [21].

\section{Conclusion}

In the present paper, we have determined some optical properties and the electronic polarizability of cubic zincblende boronantimonide semiconductor.

It is found that the experimental one (4.0 to 4.5$)$ of the reflex index reported in Ref. [10] is very higher than not only our result but also than much theoretical data reported in literature.

Using the experimental value of $\mathrm{E}_{\mathrm{g}}=0.55 \mathrm{eV}$ reported in Ref. [10], and some usual empirical formulas, the high-frequency dielectric constant was calculated and small discrepancy was observed.

The total optical electronegativity difference, the bulk modulus, the microhardness, the plasmon energy, and the electronic polarizability are also obtained. In general, our obtained results agree well with other theoretical data from the literature.

\section{References}

[1] A. S. Verma, Naresh Pal, B K Sarkar, R Bhandari, and V. K. Jindal, "Dielectric constants of zincblende semiconductors", Physica Scripta, Vol.85, No.1, (2012) pp. 015705 (4pp). and references cited therein. https://doi.org/10.1088/0031-8949/85/01/015705.

[2] D. S. Yadav, C. kumar, J. Sigh, Parashuram, and G. Kumar, "Optoelectronic properties of zinc blende and wurtzite structured binary solids", Journal of Engineering and Computer Innovations, Vol.3, No.2, (2012), pp. 26-35. http://www.academicjournals.org/journal/ JECI/article-abstract/DC4DD528645. https://doi.org/10.5897/JECI12.005.

[3] A. S. Verma, B. K. Sarkar, and V. K. Jindal, "Inherent properties of binary tetrahedral semiconductors", Physica B, Vol.45, (2010) pp. 1737-1739. https://doi.org/10.1016/j.physb.2010.01.029.

[4] B. Bouhafs, H. Aourag and M. Cartier, "Trends in band-gap pressure coefficients in boron compounds BP, BAs, and BSb", Journal of Physics: Condensed Matter, Vol. 12, No.26, (2000), pp. 56555668. https://doi.org/10.1088/0953-8984/12/26/312.

[5] S. Daoud, N. Bioud, N. Lebgaa, L. Belagraa and R. Mezouar," Pressure effect on structural, elastic and electronic properties of (B3) BSb compound ", Indian journal of physics, Vol. 87, No.4, (2013), pp. 355-362. https://link.springer.com/article/10.1007/ s12648-012-0231-y

[6] S. Daoud, " Empirical study of elastic properties of BX (X = As, $\mathrm{Sb})$ materials ", International journal of scientific world, Vol.3, No.1, (2015), pp. 37-42. https://doi.org/10.14419/ijsw.v3i1.4022.

[7] S. Daoud, "Sound velocities and thermal properties of BX (X=As, $\mathrm{Sb})$ compounds", International journal of scientific world, Vol.3, No.1, (2015), pp. 43-48. https://doi.org/10.14419/ijsw.v3i1.4039.

[8] S. Daoud, A. Bencheikh, and L. Belagraa, "Quasi-linear correlation between high-frequency and static dielectric constants in II-VI and III-V semiconductors ", International Journal of Physical Research, Vol. 5, No.1, (2014), $\quad$ pp. https://doi.org/10.14419/ijpr.v5i1.6961.

[9] Y. Yao, D. König, and M. Green, "Investigation of boron antimonide as hot carrier absorber material ", Solar Energy Materials and Solar Cells, Vol. 111, (2014), pp. 123-126. https://doi.org/10.1016/j.solmat.2012.12.029

[10] S. N. Das, R. Bhunia, S. Hussain, R. Bhar, B. R. Chakraborty, A.K. $\mathrm{Pal}$, "Synthesis and characterization of boron antimonide films by pulsed laser deposition technique", Applied Surface Science, Vol. 353, (2015), pp. 439- 448. http://dx.doi.org/10.1016/j.apsusc. 2015.06.157

[11] A. Zaoui, S. Kacimi, A. Yakoubi, B. Abbar, and B. Bouhafs, Optical properties of BP, BAs and BSb compounds under hydrostatic pressure, Physica B, Vol. 367, No. 1-4, (2005), pp. 195-204. https://doi.org/10.1016/j.physb.2005.06.018.

[12] S. Labidi, H. Meradji, S. Ghemid, S. Meçabih, B. Abbar, " Pressure dependence of electronic and optical properties of zincblende BP BAs and BSb compounds", Journal of Optoelectronics and Ad- vanced Materials, Vol. 11, No. 7, (2009), pp. 994 - 1001 http://joam.inoe.ro/index.php?option=magazine $\&$ op=view $\& i d u=19$ $89 \&$ catid $=40$.

[13] S H.A. Badehian, H. Salehi, " Ab-initio study of the structural, electronic and optical properties of BSb (110) and (100) surfaces", Surface Science, Vol.628, (2014), pp. 1-7. https://doi.org/10.1016/j.susc.2014.05.002.

[14] D. Touat, M. Ferhat and A. Zaoui, "Dynamical behaviour in the boron III-V group: a first-principles study", Journal of Physics: Condensed Matter, Vol. 18, No. 15, (2006), pp. 3647-3654. https://doi.org/10.1088/0953-8984/18/15/011.

[15] K. Bouamama, P. Djemia, N. Lebgaa and K. Kassali, "Ab initio calculation of the lattice dynamics of the Boron group-V compounds under high pressure", High Pressure Research, Vol. 27, No. 2 (2007), pp. 269 -277. http://dx.doi.org/10.1080/0895795070 1265359.

[16] S. Cui, W. Feng, H. Hu, Z. Feng, "First-principles study on the boron antimony compound", Physica Status Solidi (B), Vol. 246, No.1, (2009), pp. 119-123. http://dx.doi.org/10.1002/pssb. 200844010.

[17] R.R. Reddy, K. Rama Gopal, K. Narasimhulu, L. Siva Sankara Reddy, K. Raghavendra Kumar, G. Balakrishnaiah, and M. Ravi Kumar, "Interrelationship between structural, optical, electronic and elastic properties of materials", Journal of Alloys and Compounds, Vol. 473, (2009), pp. 28-35. http://dx.doi.org/10.1016/j.jallcom. 2008.06.037.

[18] M. Anani, C. Mathieu, S. Lebid, Y. Amar, Z. Chama and H. Abid, "Model for calculating the refractive index of a III-V semiconductor", Computational Materials Science, Vol.41, No.4, (2008), pp. 570-575. https://doi.org/10.1016/j.commatsci.2007.05.023.

[19] S. Daoud, N. Bioud, N. Lebgaa, and R. Mezouar, "Optoelectronic and thermal properties of boron- bismuth compound", International Journal of Physical Research, Vol.2, No.2, (2014), pp. 27-31. https://doi.org/10.14419/ijpr.v2i2.2760.

[20] R. R. Reddy, Y. Nazeer Ahammed, P. Abdul Azeem, K. Rama Gopal, B. Sasikala Devi, and T. V. R. Rao, "Dependence of Physica Parameters of Compound Semiconductors on Refractive Index", Defense Science Journal, Vol. 53, No. 3, (2003), pp. 239-248 http://www.publications.drdo.gov.in/ojs/index.php/dsj/article/view/ 2272. https://doi.org/10.14429/dsj.53.2272.

[21] V. P. Shaileshkumar, "Application of pseudopotential theory to certain binary, ternary and quaternary semiconductors," Thesis of Doctor of Philosophy in Physics, Sardar Patel University, India (January- 2012). http://hdl.handle.net/10603/7350.

[22] A. S. Verma, "Electronic and Optical Properties of Rare-earth Chalcogenides and Pnictides", African Physical Review, Vol.3, (2009), pp. 11-20. www.aphysrev.org/index.php/aphysrev/article/ download/.../151.

[23] S. Daoud, " Comment on: Ab initio calculations of B2 type RHg (R $=\mathrm{Ce}, \mathrm{Pr}, \mathrm{Eu}$ and $\mathrm{Gd}$ ) intermetallic compounds", The European Physical Journal B, Vol.89, (2016) 47 (2pp). https://link.springer. com /article /10.1140/epjb/e2016-60844-9.

[24] S. Daoud, "Comment on 'The effect of pressure on the physical properties of Cu3N", Physica Scripta. Vol. 91, (2016) 057001 (2pp). http://iopscience. iop.org/1402-4896/91/5/057001.

[25] S. Daoud and A. Latreche, "Comment on Density functional investigation on electronic structure and elastic properties of $\mathrm{BeX}$ at high pressure", Indian journal of physics, Vol. 90, No.11, (2016), pp. 1243-1244. http://www.readcube.com/articles/10.1007/s12648-0160863-4. https://doi.org/10.1007/s12648-016-0863-4.

[26] A. S. Verma, R. K. Singh \& S. K. Rathi, "An empirical model for dielectric constant and electronic polarizability of binary (ANB8$\mathrm{N}$ ) and ternary (ANB2+ NC2 7- N) tetrahedral semiconductors", Journal of Alloys and Compounds, Vol. 486, No. 1-2, (2009), pp. 795-800. https://doi.org/10.1016/j.jallcom.2009.07.067. 